\title{
Disorder effects on infrared reflection spectra of InN films
}

\author{
J. S. Thakur ${ }^{\mathrm{a})}$ and G. W. Auner \\ Department of Electrical and Computer Engineering, Wayne State University, Detroit, Michigan 48202 \\ D. B. Haddad and R. Naik \\ Department of Physics and Astronomy, Wayne State University, Detroit, Michigan 48201 \\ V. M. Naik \\ Department of Natural Sciences, University of Michigan-Dearborn, Dearborn, Michigan 48128
}

(Received 2 October 2003; accepted 6 February 2004)

\begin{abstract}
The effect of plasmon and LO-phonon damping on the optical measurements of InN films is discussed. Phonon and plasmon damping dramatically modifies the spectral features of the optical spectra and destabilizes the coupled modes of the system. Phonon damping affects the optical properties in a qualitatively different way from plasmon damping. Increased phonon damping leads to a merging of the coupled modes for a certain range of carrier density. Plasmon damping broadens the spectral linewidths of both of the coupled modes when plasmon energies are of the order of LO-phonon energies. However, when plasmon energies are larger than LO-phonon energies, increasing plasmon damping makes the higher-energy mode completely degenerate with plasmon, and may even have lower energies than plasmon. In weakly damped situations, we also discuss the low-energy optical transmission region that forms in between the coupled modes. Finally, the effect of plasmon and LO-phonon coupling on the analyses of the experimental infrared reflection data of InN films (for different electron densities) is discussed. (c) 2004 American Institute of Physics.
\end{abstract}

[DOI: $10.1063 / 1.1690457$ ]

\section{INTRODUCTION}

Recently there has been an extensive investigation of optical, electronic, and other properties of InN films using various experimental ${ }^{1-5}$ and theoretical techniques. ${ }^{6-9}$ These properties of the films are strongly dependent on the electron density, $n_{e}$, and disorder in the films. In spite of improved fabrication techniques, these variables (density and disorder) are still not fully controllable during the fabrication process, owing to the low dissociation temperature of the $\mathrm{InN}$ and a large lattice mismatch with the substrate. There is a stoichiometric imbalance of the nitrogen $(\mathrm{N})$ concentration in the crystal structure, which results in a broken crystal symmetry. This may lead to a modification of the vibrational modes of the lattice and may also cause their damping. A nitrogen vacancy is also known to act like a donor, ${ }^{10}$ in addition to $\mathrm{O}$ and $\mathrm{Si}$ impurities. Computations have shown that $\mathrm{H}$ could be another active donor source in $\mathrm{InN}$ semiconductor material. ${ }^{11}$ All these donors collectively generate an unknown number of electrons in the conduction band and simultaneously provide a scattering mechanism for the electrons, which leads to damping of the collective plasmon mode. Other sources of damping are negatively charged high-density threading-edge, screw, and mixed dislocations generated by the lattice mismatch. ${ }^{12,13}$ Because of these active donor sites, InN films are found to have a wide range of electron densities, ranging from $5 \times 10^{18} \mathrm{~cm}^{-3}$ to $1 \times 10^{21} \mathrm{~cm}^{-3}$, as well as a wide range of disorder that is reflected through the mobility, which varies widely from 50 to $2700 \mathrm{~cm}^{2} / \mathrm{V} \mathrm{s} .{ }^{1,2,14-17}$

\footnotetext{
a)Electronic mail: jagdish@wayne.edu
}

Optical measurements provide an efficient and reliable method to estimate the values of a wide range of physical parameters used for device characterization. ${ }^{17,18}$ These measurements also provide data about the crystal quality and surface imperfection. ${ }^{19,20}$ Most physical parameters of the film, like the dielectric constant $\varepsilon_{\infty}$, the bandgap energy $E_{g}$, the effective mass of electron $m_{e}^{*}$, the electron density $n_{e}$, mobility and crystal quality, etc., can be determined from its measured optical and electrical characteristics. Recently, the photoluminescence spectra of single crystalline InN films showed that the $E_{g}$ value of InN films is much smaller than the generally accepted value of $1.9 \mathrm{eV}$. The smaller reported values found in the range of $0.65-1.0 \mathrm{eV}^{1,2}$ seem to be associated with improvements in the thin film fabrication techniques leading to higher quality $\mathrm{InN}$ films with relatively less disorder. Since these measurements depend on $n_{e}$ and the level of disorder in the system, it is important to discuss how these parameters ( $n_{e}$ and disorder) influence the optical measurements.

The behavior of optical properties is directly linked to the collective modes-fundamental or coupled modes-of the system. Because of the polar nature of $\mathrm{InN}$ film, the plasmon interaction with LO phonons creates two coupled modes: ${ }^{21} \omega^{+}$, the higher-energy coupled mode and, $\omega^{-}$, the lower-energy coupled mode. These modes are directly associated with the reflection edges of the optical reflection spectra, and are also expected in the Raman spectra if they are not damped. However, unambiguous identification of these modes is often difficult due to their strong dependence on plasmon and LO phonon damping, and other physical properties of the system. These not only affect the intensity of 
spectral lines but also shift the spectral-peak positions associated with these modes. In samples where the electron density is such that the plasmon energy is comparable to LO phonon energy, stronger phonon damping can even lead to the merging of the two coupled-modes into a single mode. At higher electron densities, the weaker coupling between the large-energy plasmon and the LO phonon again makes the identification of these modes difficult. At lower densities, thermal effects produce additional broadening of spectral lines and have a pronounced effect on the spectral properties.

In this paper we discuss the instability of the coupledmodes against damping of the fundamental modes- the plasmon and the LO phonon-over a wide range of electron densities. We analyze the effect on their dispersions and hence how the corresponding measurable spectral features are affected by such damping.

\section{DESCRIPTION OF COUPLED PLASMON-PHONON MODES}

The behavior of the optical properties, like the reflection edge and Raman spectra, etc., can be analyzed from the spectral line-shape function $\operatorname{Im}(1 / \varepsilon(\omega))$. Here, $\varepsilon(\omega)$ is the long wavelength limit of the total dielectric function for the phonon and plasmon system, which can be described using a well-known linear response model: $:^{22,23}$

$$
\varepsilon(\omega)=\varepsilon_{\infty}\left[1+\frac{\left(\omega_{\mathrm{LO}}^{2}-\omega_{\mathrm{TO}}^{2}\right)}{\left(\omega_{\mathrm{TO}}^{2}-\omega^{2}-i \omega \gamma\right)}-\frac{\omega_{p}^{2}}{\left(\omega^{2}+i \omega \Gamma\right)}\right],
$$

where $\omega_{p} / \Gamma, \omega_{\mathrm{LO}} / \gamma$, and $\omega_{\mathrm{TO}}$ are the plasmon, LO-phonon resonant-frequency/damping parameters, and TO-phonon frequency, respectively. The contribution to the optical intensity from the single particle-hole pair excitations vanishes in the long wavelength limit and is not discussed here. LO- and TO-phonon frequencies are taken from the Raman spectra of InN films, and their values are 590 and $450 \mathrm{~cm}^{-1}$, respectively. ${ }^{24,25}$

The deviation in the energy of the coupled modes from the fundamental modes depends directly on the plasmon and LO-phonon energies. In the extreme limits of $\omega_{p} \ll \omega_{\text {LO }}$ or $\omega_{p} \gg \omega_{\mathrm{LO}}$, the coupling between the fundamental modes is very weak and their spectral features become similar to those of the fundamental modes. Overdamping of a particular mode can also lead to a weak coupling situation. However, in between the above limiting regions, there is a wide region of coupling where none of the fundamental modes dominates and the Raman spectra, optical properties, and dispersions of the coupled modes etc. become nontrivial functions of the fundamental mode energies and their damping rates.

Figure 1(a) shows a three-dimensional plot of $\operatorname{Im}(1 / \varepsilon(\omega))$ as a function of frequency and of plasmon energy. In a weakly disordered system, the spectral lines associated with coupled modes are well defined. However, the spectral intensity associated with the $\omega^{-}$mode decays rapidly in both higher and lower electron density regimes [Fig. 1(b)]. The wide valley in between these coupled modes represents an optical transmission region, if there is no other absorption process in the system. The width of this low-
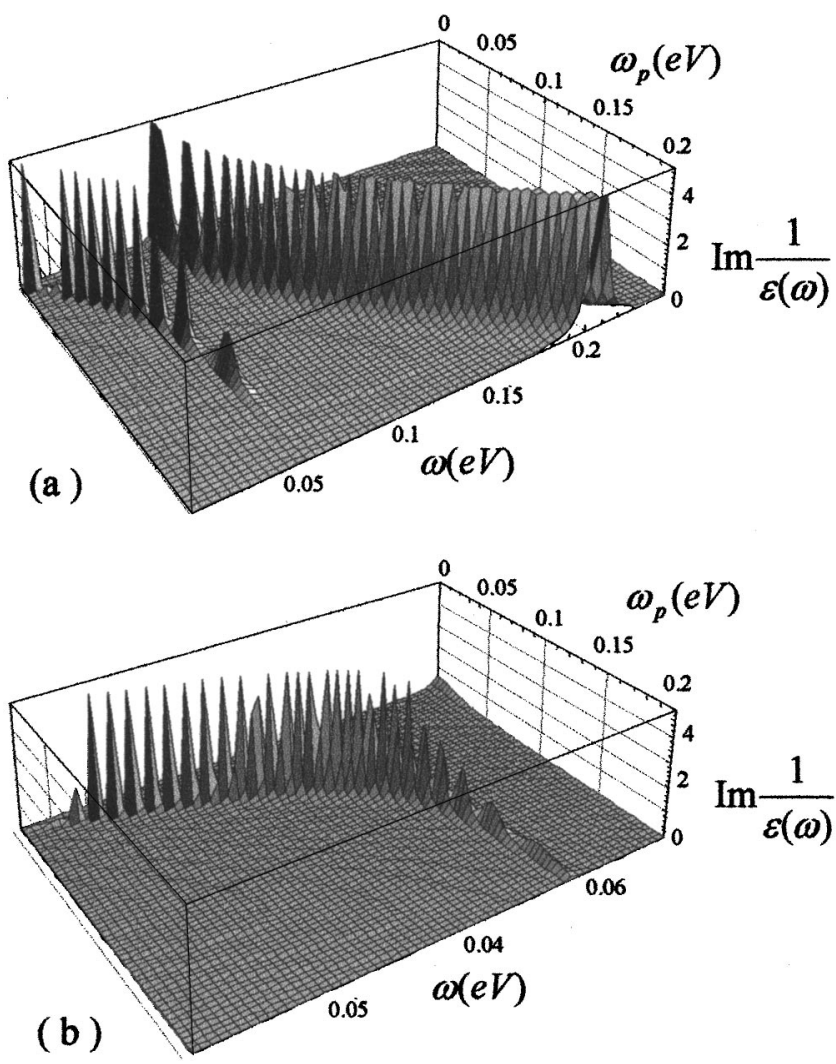

FIG. 1. (a) Three-dimensional plot of $\operatorname{Im}(1 / \varepsilon(\omega))$ as a function of $\omega_{p}$ and $\omega$ for $\gamma=0.01 \omega_{\mathrm{LO}}$ and $\Gamma=0.01 \omega_{p}$; (b) showing only the $\omega^{-}$mode with the same parameters as that of (a).

energy transmission region depends on the energy separation between the plasmon and phonon frequencies. It is given by

$$
T_{\text {low }}=\omega^{+}-\omega^{-} \text {. }
$$

Of course, this width is very sensitive to the damping of the fundamental modes, which will be discussed in later sections of this paper. In the low electron-density limit, $T_{\text {low }}$ reduces to a constant value $\sqrt{2} \omega_{\mathrm{LO}}$ (independent of electron density), but in the high-density regime, it becomes wider and increases with plasmon energy as $\sqrt{2} \omega_{p}$.

A much-extended region of optical transmission exists between the upper reflection edge $\omega^{+}$and the direct bandabsorption edge, whose width depends on the bandgap value and the chemical potential of the material. This region is given by

$$
T_{\text {high }}=\left(E_{g}+m_{v} \zeta\right)-\omega^{+},
$$

where $E_{g}$ is the direct bandgap energy, $m_{v}=m_{e}^{*} / m_{h}^{*}+1$ and $\zeta$ is the chemical potential of the disordered system. ${ }^{24}$ Both disorder and carrier density strongly affect this region through $\zeta$ and $\omega^{+}$.

Sensitivity of the optical properties to the plasmon and phonon damping depends on the coupling between the fundamental modes. The coupling can be tuned by varying the electron density at fixed values of other parameters, thus producing a progressive transition from weakly to strongly coupled modes. The whole range of electron density variation can be divided into three distinct regions where the be- 
havior of the coupled modes is characteristically different with respect to the variation of damping parameters. These regions are

$$
\begin{aligned}
& \text { I } \omega_{p}<\frac{\omega_{\mathrm{LO}}}{2}, \\
& \text { II } \frac{\omega_{\mathrm{LO}}}{2}<\omega_{p}<2 \omega_{\mathrm{LO}}, \\
& \text { III } \omega_{p}>2 \omega_{\mathrm{LO}} .
\end{aligned}
$$

Regions I and III represent weak coupling, where the spectral features of a particular coupled mode are determined mainly by the parameters of the fundamental mode. By contrast, in region II the two fundamental modes are strongly coupled.

In region I, the $\omega^{-}$mode dispersion varies linearly with $\omega_{p}$; for small damping, the spectral lines of the modes remain sharply peaked. On the other hand, the dispersion of $\omega^{+}$mode is almost independent of $\omega_{p}$ and approaches the $\omega_{\mathrm{LO}}$ mode from above. The spectral intensity of this mode does not vary appreciably with increasing $\omega_{p}$. However, it is much larger than that of the $\omega^{-}$mode. At such low levels of disorder, both the modes remain well separated and well defined. Even though the corresponding electron density $n_{e}$ $<\left(\omega_{\mathrm{LO}} / 2\right)^{2}\left(\varepsilon_{\infty} m_{e}^{*} / 4 \pi e^{2}\right)<6.7 \times 10^{17} \mathrm{~cm}^{-3}$ is relatively small in this region, it has become possible to fabricate samples with less disorder and lower electron density due to improved fabrication techniques.

In region II, $6.7 \times 10^{17} \mathrm{~cm}^{-3}<n_{e}<1.6 \times 10^{19} \mathrm{~cm}^{-3}$, both the modes are strongly coupled. In this region, a change in either mode's parameters affects the spectral features of both the coupled modes significantly. The energy of the coupled modes increases continuously with increasing value of the plasmon energy.

In region III, where $n_{e}>1.6 \times 10^{19} \mathrm{~cm}^{-3}$, the energy of the $\omega^{+}$mode begins to increase linearly with $\omega_{p}$ and that of $\omega^{-}$mode now becomes almost independent of $\omega_{p}$ and approaches $\omega_{\text {TO }}$ from below. At this level of electron concentration, the coupling between the fundamental modes is weak due to the large difference in plasmon and LO-phonon energies. The spectral features of the coupled modes $\omega^{+}$and $\omega^{-}$ become similar to those of the plasmon and TO modes, respectively. Thus, if there is appreciable disorder in the system, the coupled modes' unambiguous identification as distinct from the fundamental modes becomes an experimental challenge. This difficulty is further enhanced in the case of the $\omega^{-}$coupled mode, because of a rapid decrease in its spectral intensity at higher values of electron density.

\section{PHONON DAMPING}

Disorder in a system plays a key role in the destabilization of the coupled modes. Figures 2(a) and 2(b) show the behavior of the coupled modes with increasing levels of $\gamma$. We have taken a fixed value of $\Gamma=0.2 \omega_{p}$, which approximately represents the disorder level for electron scattering in films. We see from Figs. 1(a) and 2(a) that, as the plasmon damping is tuned up from $0.01 \omega_{p}$ to $0.2 \omega_{p}$, there are significant modulations in the spectral features of both the
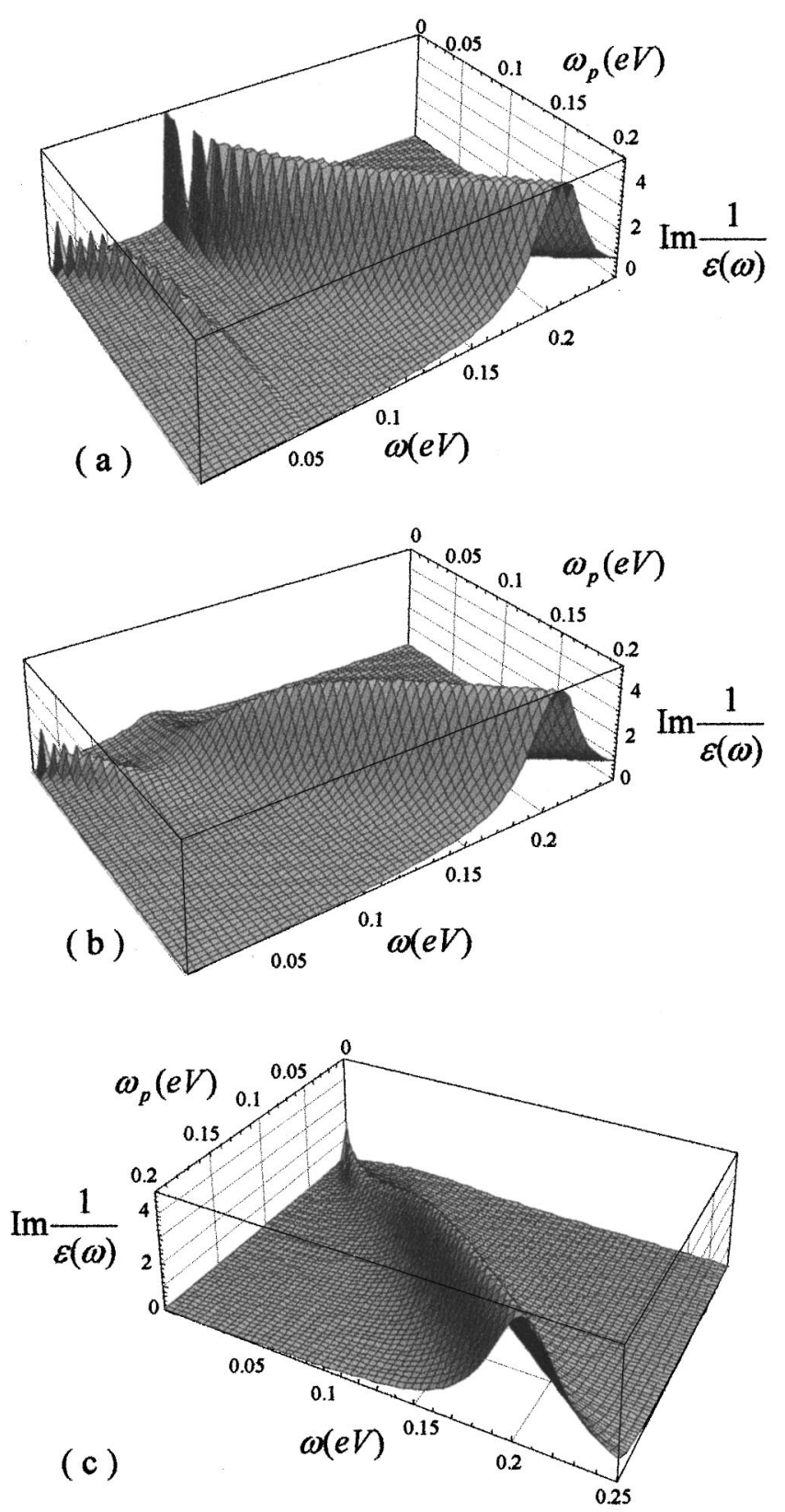

FIG. 2. Three-dimensional plot of $\operatorname{Im}(1 / \varepsilon(\omega))$ as a function of $\omega_{p}$ and $\omega$ for (a) $\Gamma=0.2 \omega_{p}$ and $\gamma=0.01 \omega_{\mathrm{LO}}$; (b) $\Gamma=0.2 \omega_{p}$ and $\gamma=0.5 \omega_{\mathrm{LO}}$; (c) $\Gamma$ $=0.2 \omega_{p}$ and $\gamma=1.2 \omega_{\mathrm{LO}}$.

coupled modes. For example, the spectral intensity of the $\omega^{-}$ mode sharply decreases with increasing $\omega_{p}$. As $\gamma$ increases, the linewidth of the $\omega^{+}$mode in the lower electron-density region gradually broadens and a similar broadening happens to the $\omega^{-}$mode in the higher electron-density region. This is because the character of the coupled modes in these regions is mainly determined by the LO phonon. As $\gamma$ increases further, the $\omega^{-}$mode starts to disappear from the higher plasmon-energy region, while $\omega^{+}$begins to broaden and loses its spectral strength on the lower-energy side. In the reflection spectra, the reflection edge associated with the $\omega^{-}$ mode will smear out, resulting in transmission down toward the zero-energy region, if there is no other absorption process there. When $\gamma \approx \omega_{\mathrm{LO}}$, Fig. 2(c), the two regions where $\omega^{+}$ 

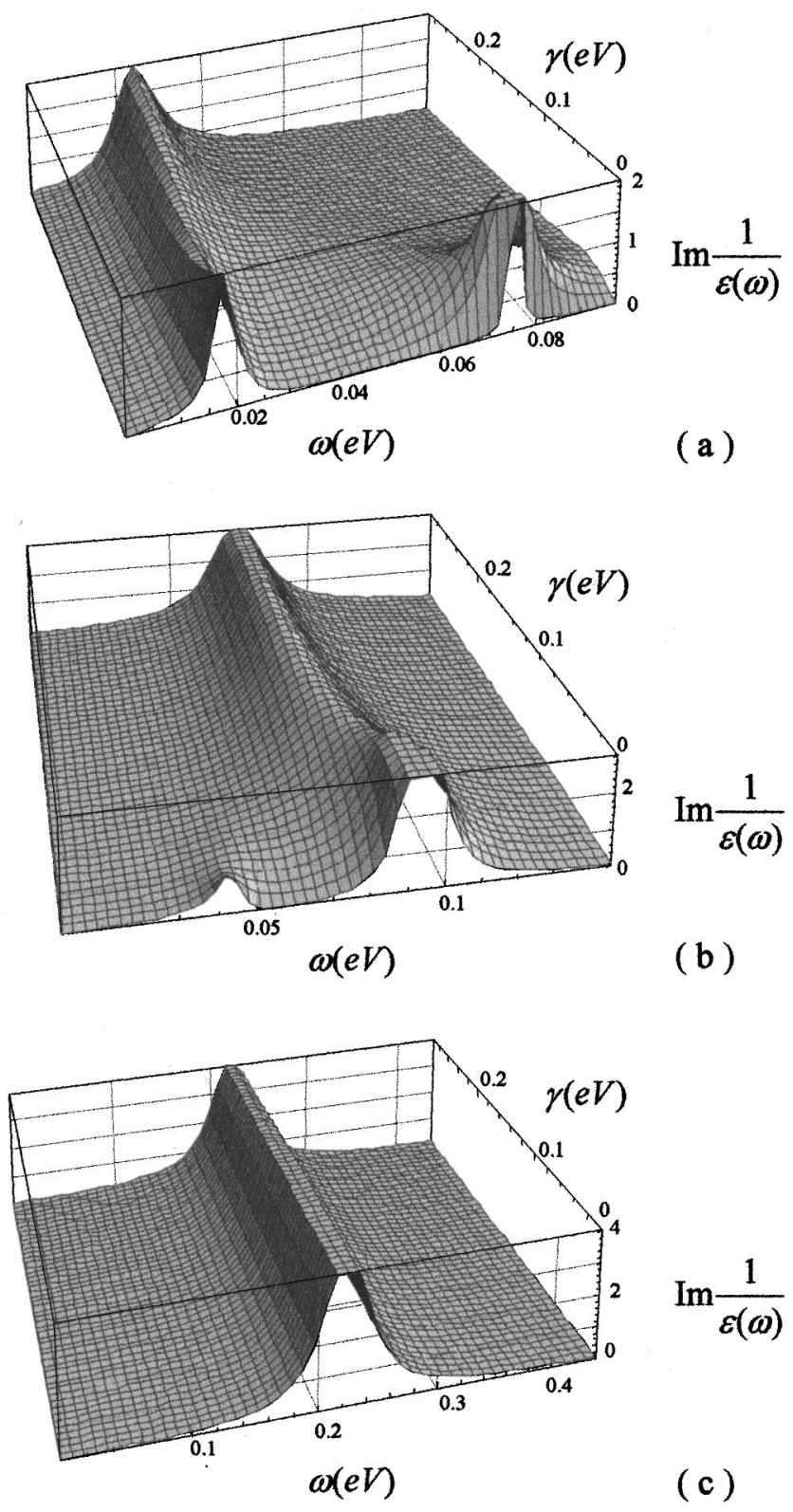

FIG. 3. Three-dimensional plot of $\operatorname{Im}(1 / \varepsilon(\omega))$ as a function of $\gamma$ and $\omega$ for $\Gamma=0.2 \omega_{p}$ and (a) $\omega_{p}=\omega_{\mathrm{LO}} / 3$, (b) $\omega_{p}=\omega_{\mathrm{LO}}$, and (c) $\omega_{p}=3 \omega_{\mathrm{LO}}$.

and $\omega^{-}$were independent of $n_{e}$ disappear and the two coupled modes merge to form a single mode and then persist as a single mode over the whole range of $n_{e}$. The energy of this single mode varies linearly with plasmon energy over the whole range of electron density.

In the following we discuss the shift in the peak positions of the coupled modes with increasing phonon damping in all the three regions: I, II, and III. Figure 3(a) shows the effects of increasing $\gamma$ on the spectral features of the coupled modes when $n_{e}=2.9 \times 10^{17} \mathrm{~cm}^{-3}$ or $\omega_{p}=\omega_{\mathrm{LO}} / 3$, and $\Gamma$ $=0.2 \omega_{p}$. In this low- $n_{e}$ regime (region $\mathrm{I}$ ), the $\omega^{+}$mode damps rapidly with increasing phonon damping, but its peak position does not shift until $\gamma$ becomes less than $0.3 \omega_{\mathrm{LO}}$. However, by the time $\gamma$ becomes $\approx \omega_{\mathrm{LO}}$, its peak position has moved from $0.075 \mathrm{eV}$ (at $\gamma=0.02 \omega_{\mathrm{LO}}$ ) to $0.061 \mathrm{eV}$. At the same time the $\omega^{-}$mode remains unchanged over the whole range of $\gamma$ variation shown in the figure, except for a slight upward shift in the peak position at $\gamma=\omega_{\mathrm{LO}}$. The intensity of this mode slowly decreases with increasing values of $\gamma$. The precursor of the merging phenomenon of the coupled modes can be seen at this low electron density.

In contrast to the plasmon damping, which shifts the peak positions toward lower values, the variation in phonon damping induces qualitatively different effects on the optical properties. Particularly in region II, an increase in phonon damping shifts the peak position in the opposite direction. Figure 3(b) shows the effects of increasing $\gamma$ when $\omega_{p}$ $=\omega_{\mathrm{LO}}\left(n_{e}=2.6 \times 10^{18} \mathrm{~cm}^{-3}\right)$ at a fixed $\Gamma=0.2 \omega_{p}$. With increasing values of $\gamma$, the peak positions of the $\omega^{-}$mode shift towards higher energies and the spectral intensities decrease rapidly with $\gamma$. On the other hand, the peak position of the $\omega^{+}$mode, as well as its intensity, decrease with increasing $\gamma$. As $\gamma$ increases further, both the modes begin to merge and form a single mode. In region II, the two coupled modes come close to each other (they are closest when $\omega_{p}=\omega_{\mathrm{TO}}$ ), and if there is a damping effect, it can quickly induce them to overlap.

Figure 3(c) is a representative example from region III with $\omega_{p}=3 \omega_{\mathrm{LO}} \quad\left(n_{e}=2.8 \times 10^{19} \mathrm{~cm}^{-3}\right)$, and $\Gamma=0.2 \omega_{p}$. When the electron density becomes large, the intensity of both the coupled modes decreases with increasing $\gamma$. The peak position of the $\omega^{+}$mode remains fixed over the range of variation of $\gamma$ shown in Fig. 3(c). However, the intensity of the $\omega^{-}$mode decreases much more rapidly with increasing $\gamma$, and for $\gamma>0.2 \omega_{\mathrm{LO}}$ its peak structure completely disappears.

\section{PLASMON DAMPING}

The locations of the peak positions of the spectral lines not only depend on the fundamental mode energies but also on their damping. Increased plasmon damping shifts the peak position of its spectral line toward the lower-energy side of $\omega_{p}$. But when the fundamental modes are coupled, the direction of the shift in peak positions, from their corresponding undamped values, depends on the ratio $\omega_{p} / \omega_{\mathrm{LO}}$. In Figs. 4(a)-4(c), we show three-dimensional plots of $\operatorname{Im}(1 / \varepsilon(\omega))$ as functions of $\omega_{p}$ and $\omega$ for various values of plasmon damping at a fixed and small value of $\gamma=0.2 \omega_{\mathrm{LO}}$. At $\Gamma$ $=0.01 \omega_{p}$, the broadening effects on the $\omega^{+}$mode in its lower-energy region and on the $\omega^{-}$mode on its higherenergy region arise from phonon damping $\left(\gamma=0.2 \omega_{\mathrm{LO}}\right)$. As $\Gamma$ increases further, there is a substantial broadening in the spectral intensity of the $\omega^{-}$mode. This is due to the fact that the lower-energy region of this mode is associated with the plasmon and hence its spectral intensity is directly affected by plasmon damping. In the intermediate plasmon energy region, both the coupled modes are affected by plasmon damping due to the strong coupling of the fundamental modes. On the other hand, the spectral intensity associated with $\omega^{+}$begins to broaden only in the higher-energy region. As $\Gamma$ increases further, the broadening of the $\omega^{+}$mode spreads into region II, while its spectral features remain unaltered on the lower-energy side due to its association with the $\omega_{\mathrm{LO}}$ mode. 

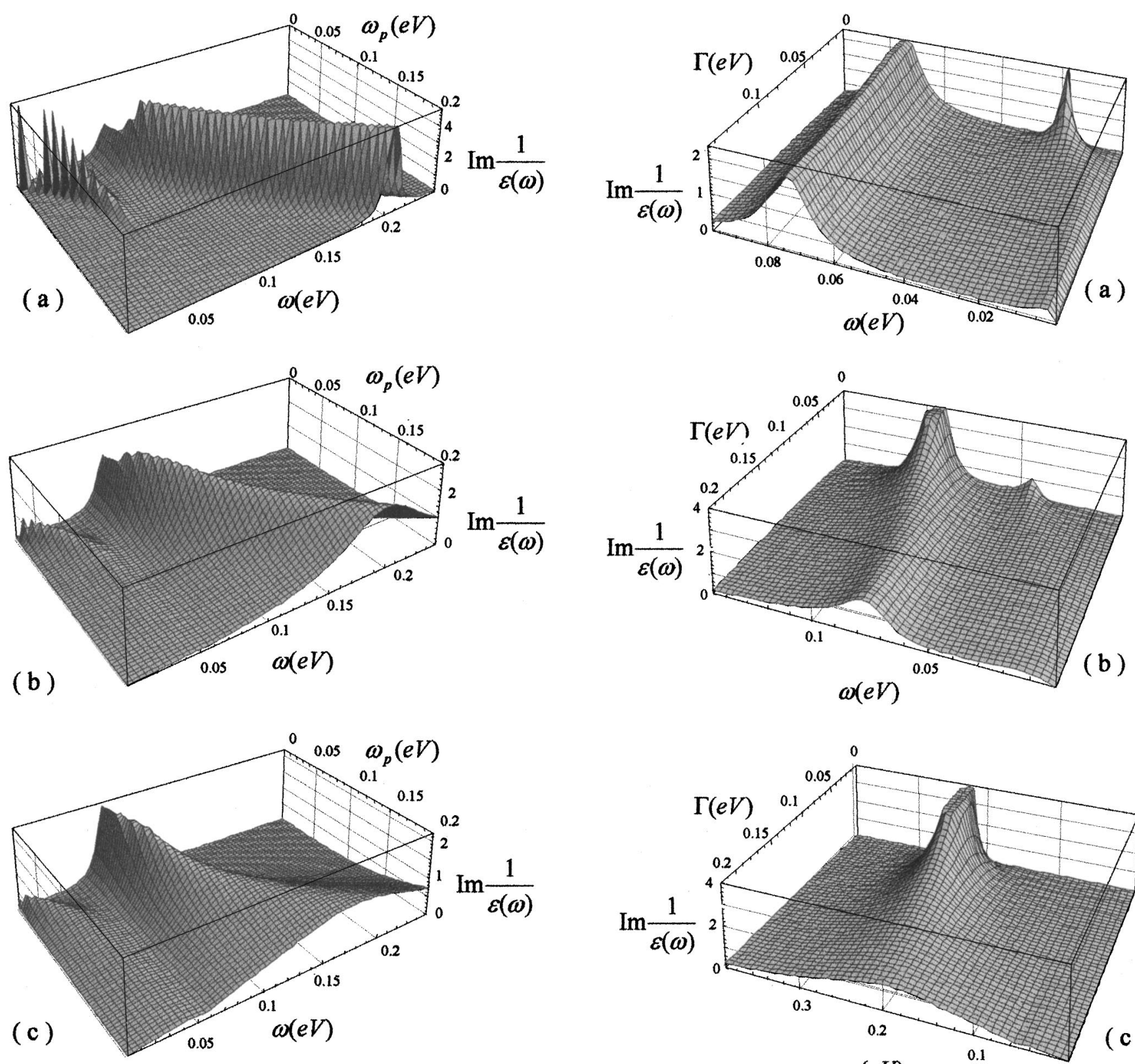

FIG. 4. Three-dimensional plot of $\operatorname{Im}(1 / \varepsilon(\omega))$ as a function of $\omega_{p}$ and $\omega$ for $\gamma=0.2 \omega_{\mathrm{LO}}$ and (a) $\Gamma=0.01 \omega_{p}$, (b) $\Gamma=0.5 \omega_{p}$, and (c) $\Gamma=\omega_{p}$.

In Figs. 5(a)-5(c) we show how the peak positions of the coupled modes shift with increasing plasmon damping. Figure 5(a) shows $\operatorname{Im}(1 / \varepsilon(\omega))$ as a function of $\Gamma$ and $\omega$ for the fixed value of $\gamma=0.2 \omega_{\mathrm{LO}}=120 \mathrm{~cm}^{-1}$ and electron density $n_{e}=2.9 \times 10^{17} \mathrm{~cm}^{-3}\left(\omega_{p}=\omega_{\mathrm{LO}} / 3\right)$. Although the value of $\gamma$ used here is somewhat large, it is not an uncommon one in poor quality InN films. In region I, one expects no significant shift in the peak position of $\omega^{+}$as $\Gamma$ increases. However, the peak position for $\omega^{-}$shifts significantly away from the plasmon mode. In this range of $n_{e}$, the character of the $\omega^{-}$mode is similar to that of the plasmon, where charges are screened with an essentially static screening factor. However, its identification with the plasmon will lead to an underestimation of the plasmon energy. This mode also becomes strongly damped with increasing $\Gamma$. We expect these effects

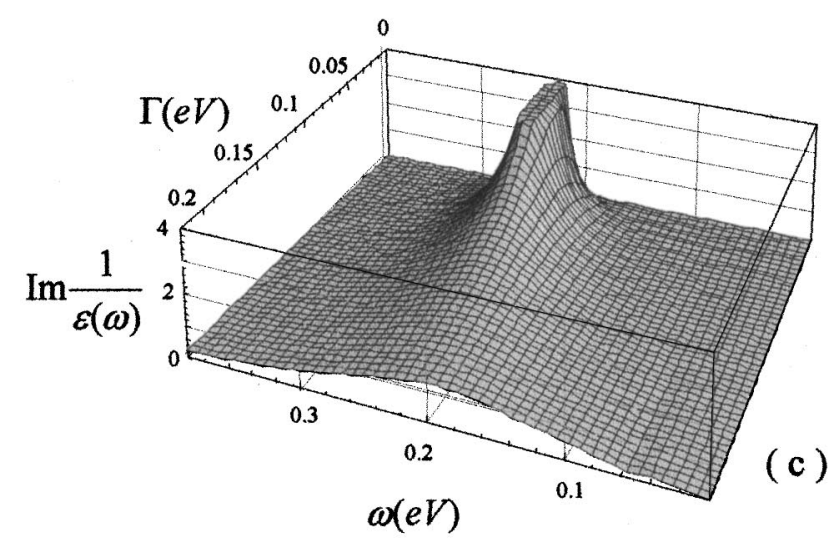

FIG. 5. Three-dimensional plot of $\operatorname{Im}(1 / \varepsilon(\omega))$ as a function of $\Gamma$ and $\omega$ for $\gamma=0.2 \omega_{\mathrm{LO}}$ and (a) $\omega_{p}=\omega_{\mathrm{LO}} / 3$, (b) $\omega_{p}=\omega_{\mathrm{LO}}$, and (c) $\omega_{p}=3 \omega_{\mathrm{LO}}$.

to be more pronounced at lower values of phonon damping.

In Fig. 5(b), we have $n_{e}=2.6 \times 10^{18} \mathrm{~cm}^{-3}\left(\omega_{p}=\omega_{\mathrm{LO}}\right)$, and $\gamma=0.2 \omega_{\mathrm{LO}}$. In this region, the peak positions of both the modes move downward substantially with increasing plasmon damping. For example, the peak position of the $\omega^{-}$ mode shifts from 0.044 to $0.014 \mathrm{eV}$ over the range of $\Gamma$ shown in the figure. On the other hand, the peak position of the $\omega^{+}$mode moves from 0.093 to $0.077 \mathrm{eV}$ over the same range of $\Gamma$. In this region of $n_{e}$, the modes are strongly coupled, and hence their peak positions become sensitive to the plasmon damping. The estimated values of the peak positions are smaller than their corresponding undamped resonant values.

Figure 5(c) shows a typical example from region III with $n_{e}=2.8 \times 10^{19} \mathrm{~cm}^{-3}\left(\omega_{p}=3 \omega_{\mathrm{LO}}\right)$ and $\gamma=0.2 \omega_{\mathrm{LO}}$. In this 
region, the decrease in the peak position of the $\omega^{+}$mode is comparatively more pronounced when plasmon damping becomes large. The peak position of this mode decreases from 0.23 to $0.19 \mathrm{eV}$ over the range of $\Gamma$ shown in the figure. Note that over this range of plasmon damping, the $\omega^{+}$energy crosses the fundamental plasmon mode $\left(\omega_{p}=0.219 \mathrm{eV}\right)$ energy, and even becomes much smaller than $\omega_{p}$. This is contrary to its expected behavior, for one expects it to stay on the higher-energy side of the plasmon mode. At such levels of damping, it is difficult to distinguish experimentally between the fundamental and coupled modes as qualitatively separate excitations, especially when the $\omega^{+}$mode becomes completely degenerate with the plasmon. On the other hand, the peak position of the $\omega^{-}$mode decreases from 0.056 to $0.043 \mathrm{eV}$ over the same range of $\Gamma$.

Normally, in the analysis of experimental data, the highenergy peak position is equated to $\omega_{p}$ to estimate the value of a physical parameter such as the electron density, $n_{e}$ or effective mass, $m_{e}^{*}$, etc. of the system. This can lead to a substantial error in the estimated value of the parameters, if the damping effects are strong and not incorporated into the calculations. Arriving at a reliable measure of the damping corrections is therefore of considerable practical importance.

\section{EXPERIMENTAL INFRARED REFLECTION SPECTRA OF InN FILMS}

Infrared reflection spectra have been reported for highquality wurtzite $\mathrm{InN}$ (0001) films ${ }^{26}$ with a range of freeelectron concentrations $\left(5 \times 10^{18}-5 \times 10^{19} \mathrm{~cm}^{-3}\right)$ as well as for polycrystalline InN films ${ }^{27}$ with $n_{e} \sim 5 \times 10^{20} \mathrm{~cm}^{-3}$. The later InN films (thickness $\sim 0.5 \mu \mathrm{m}$ ) were deposited on (0001) sapphire by a plasma-source molecular beam epitaxy (PSMBE) technique ${ }^{28}$ utilizing a hollow cathode source lined with MBE grade indium. Base pressure of the growth chamber was $\sim 10^{-8}$ Torr. A substrate temperature in the range of $325-475{ }^{\circ} \mathrm{C}$ was maintained during the growth of the films. The substrate was preheated to $800^{\circ} \mathrm{C}$ for $1 \mathrm{~h}$ and then cooled down to $325^{\circ} \mathrm{C}-475^{\circ} \mathrm{C}$ before the deposition of films.

$\theta-2 \theta$ x-ray diffraction scans indicated that films grown at $475{ }^{\circ} \mathrm{C}$ were completely $c$-axis textured whereas film growth at $325^{\circ} \mathrm{C}$ resulted in polycrystalline films. Hall effect measurements using the Van der Pauw method showed both films to be $n$-type with carrier concentrations of (3.0 \pm 0.6$) \times 10^{20} \mathrm{~cm}^{-3}$ for the $475^{\circ} \mathrm{C}$ grown film and $(8.0$ $\pm 1.6) \times 10^{20} \mathrm{~cm}^{-3}$ for the $325^{\circ} \mathrm{C}$ grown film.

Optical reflectance spectra measured for these InN films are shown in Fig. 6. The strong reflection edge observed at low energy for both films is associated with the plasmon resonance in the films. In the above studies, ${ }^{26,27}$ the reflection edge was fitted using a standard dielectric function that included only finite lifetime broadening effects for the plasmon but no phonon coupling effects [i.e., the second term in Eq. (1) is zero]. The fitted parameters, $\varepsilon_{\infty}, \omega_{p}$, and $\Gamma$ are listed in Table I, along with $n_{e}$ determined from Hall measurements. The high carrier concentrations observed in these films could result from the presence of defects stemming from either nitrogen vacancies and/or other impurities (oxy-

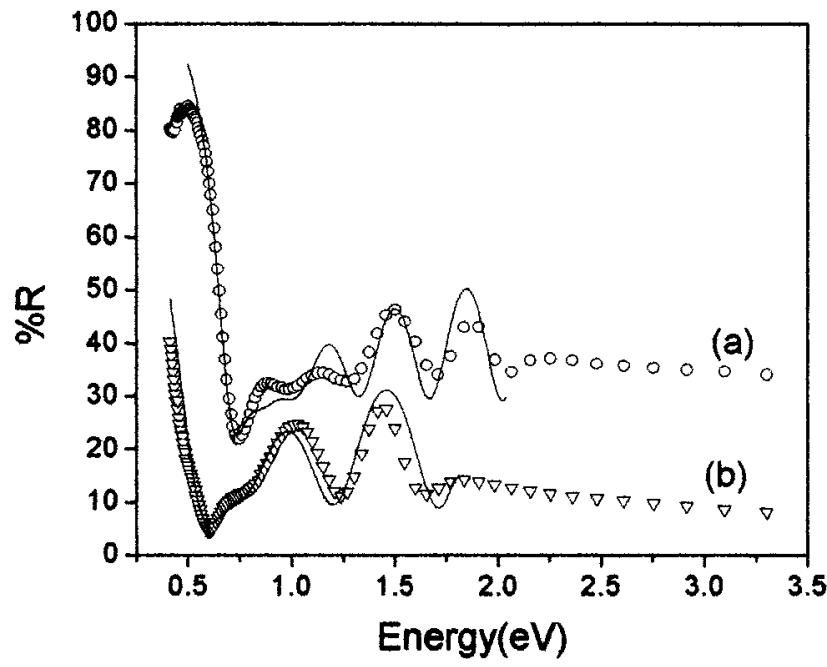

FIG. 6. Reflectance spectra measured for InN films grown at $325^{\circ} \mathrm{C}$ (a) and $475^{\circ} \mathrm{C}$ (b). Theoretical fits to the reflectance are shown by the solid lines below the bandgap absorption. Spectrum (a) is vertically offset by $20 \%$.

gen) that are typically found in InN films. ${ }^{10}$ Roomtemperature Hall mobility was determined to be $\sim 16$ $\mathrm{cm}^{2} / \mathrm{V} \mathrm{s} \quad\left(\sim 55 \mathrm{~cm}^{2} / \mathrm{V} \mathrm{s}\right)$ for the films with $n_{e}$ $=8 \times 10^{20} \mathrm{~cm}^{-3}\left(3 \times 10^{20} \mathrm{~cm}^{-3}\right)$. The effective mass $\left(m_{e}^{*}\right)$ shown in Table I was calculated using the relation $m_{e}^{*}$ $=4 \pi n_{e} e^{2} / \varepsilon_{\infty} \omega_{p}^{2}$, which monotonically increases with carrier density. The carrier density dependence of $m_{e}^{*}$ has been attributed to the nonparabolic behavior of the conduction band due to $\mathbf{k} \cdot \mathbf{p}$ interaction effects. ${ }^{26}$ Assuming that the main contribution to the mobility arises from the ionized impurity scattering centers, we estimate the number of these ionized center using the well-known Brooks-Herring ${ }^{29}$ equation. In Table I we show the values of ionized impurity concentration $N_{i}\left(\mathrm{~cm}^{-3}\right)$ for each film using corresponding values for $m_{e}^{*}$ and $\varepsilon_{\infty}$.

To investigate the effect of plasmon-phonon coupling in these films, the reflectance data have been analyzed using Eq. (1). The values of phonon energies- $\mathrm{A}_{1}(\mathrm{TO})$ phonon $\left(\omega_{\mathrm{TO}} \sim 450 \mathrm{~cm}^{-1}\right)$ and unscreened $\mathrm{A}_{1}(\mathrm{LO})\left(\omega_{\mathrm{LO}}\right.$ $\sim 590 \mathrm{~cm}^{-1}$ ) - have been taken from Raman spectra ${ }^{24,25}$ and a typically observed value of $\gamma_{\mathrm{LO}} \sim 30 \mathrm{~cm}^{-1}$ is used in Eq. (1) to fit the experimental data. The parameter $\Gamma$ is determined in such a way that the shape of the reflectance data at the reflectance edge is reproduced for each sample. The new values of $\omega_{p}, \Gamma$ and also $m_{e}^{*}$ are shown in Table I. Clearly, there is a significant shift in the values of $\omega_{p}$ for samples 1 (shift $105 \mathrm{~cm}^{-1}$ ) and 2 (shift $70 \mathrm{~cm}^{-1}$ ) compared to samples 4 and 5. This is due to the fact that samples 1 and 2 fall in region II $\left(\omega_{\mathrm{LO}} / 2<\omega_{p}<2 \omega_{\mathrm{LO}}\right)$, where modes are strongly coupled, whereas samples 4 and 5 belong to the weakly coupled region III $\left(\omega_{p} \gg 2 \omega_{\mathrm{LO}}\right)$, where the shift in the values of plasmon energy is not very large. The energy of the plasmon for sample 3 lies above the lower edge of the region III where coupling effects are not significant. Finally, the calculated values of the $\omega^{-}$coupled mode are also listed in Table I, although no experimental values are available in this frequency region for comparison. 
TABLE I. Fitted parameters for various InN samples. ${ }^{a}$

\begin{tabular}{|c|c|c|c|c|c|c|c|c|c|}
\hline \multirow{2}{*}{$\begin{array}{c}n_{e}\left(\mathrm{~cm}^{-3}\right) \\
\text { (Hall Effect) }\end{array}$} & \multirow[b]{2}{*}{$\varepsilon_{\infty}$} & \multicolumn{3}{|c|}{ Neglecting phonon coupling in Eq. (1) } & \multicolumn{5}{|c|}{ Including phonon coupling in Eq. (1) } \\
\hline & & $\omega_{p}\left(\mathrm{~cm}^{-1}\right)$ & $\Gamma\left(\mathrm{cm}^{-1}\right)$ & $m_{e}^{*} / m_{e}$ & $\omega_{p}\left(\mathrm{~cm}^{-1}\right)$ & $\Gamma\left(\mathrm{cm}^{-1}\right)$ & $N_{i}\left(\mathrm{~cm}^{-3}\right)$ & $m_{e}^{*} / m_{e}$ & $\omega^{-}\left(\mathrm{cm}^{-1}\right)^{\mathrm{b}}$ \\
\hline $5.5 \times 10^{18}$ & 6.7 & 950 & 100 & 0.082 & 845 & 117 & $4.7 \times 10^{18}$ & 0.103 & 408 \\
\hline $1.2 \times 10^{19}$ & 6.7 & 1240 & 60 & 0.104 & 1170 & 64 & $4.9 \times 10^{18}$ & 0.117 & 429 \\
\hline $4.5 \times 10^{19}$ & 6.7 & 1980 & 250 & 0.154 & 1940 & 260 & $3.2 \times 10^{19}$ & 0.160 & 435 \\
\hline $3.0 \times 10^{20}$ & 7.5 & 4114 & 1370 & 0.212 & 4100 & 1382 & $5.4 \times 10^{21}$ & 0.213 & $\ldots$ \\
\hline $8.0 \times 10^{20}$ & 7.5 & 5485 & 1048 & 0.318 & 5480 & 1054 & $4.6 \times 10^{22}$ & 0.319 & $\ldots$ \\
\hline
\end{tabular}

${ }^{a}$ See Ref. 26 for samples $1-3$ and Ref. 24 for samples 4 and 5 .

${ }^{\mathrm{b}}$ For samples 4 and $5 \omega^{-}$mode has negligible intensity.

\section{SUMMARY}

We have discussed the role of plasmon and LO-phonon damping on the infrared reflection spectra of InN films. In addition to the generic expected broadening of the spectral lines induced by damping, the phonon damping can lead to the merging of the coupled modes into a single mode. The sensitivity of the spectral lines associated with the coupled modes, to the damping of the plasmon or phonon depends on the ratio $\omega_{p} / \omega_{\mathrm{LO}}$. Strong plasmon or phonon damping can lead to an ambiguous assignment of the coupled modes. The spectral intensity of the $\omega^{-}$mode is strongly dependent on the damping and the electron density of the system. The structure of this mode diminishes rapidly in both the weakly coupled regime (high and low electron density). This renders its experimental detection a nontrivial problem. Our analysis of the experimental infrared reflection data of $\mathrm{InN}$ films shows that the plasmon-LO-phonon coupling is not negligible for films with $n_{e} \sim 5 \times 10^{18}-5 \times 10^{19} \mathrm{~cm}^{-3}$.

\section{ACKNOWLEDGMENTS}

This work was supported by NSF-IGERT-DGE Grant No. 9870720 and by the Center for Smart Sensors and Integrated Microsystems at Wayne Sate University.

${ }^{1}$ V. Yu Davydov et al., Phys. Status Solidi B 229, R1 (2002); 230, R4 (2002).

${ }^{2}$ J. Wu, W. Walukiewicz, K. M. Yu, J. W. Ager, III, E. E. Haller, H. Lu, W. J. Schaff, Y. Saito, and Y. Nanishi, Appl. Phys. Lett. 80, 3967 (2002).

${ }^{3}$ T. Matsuoka, H. Okamoto, M. Nakao, H. Harima, and E. Kurimoto, Appl. Phys. Lett. 81, 1246 (2002).

${ }^{4}$ G. Kaczmarczyk, A. Kaschner, S. Reich, A. Hoffmann, C. Thomsen, D. J. As, A. P. Lima, D. Schikora, K. Lischka, R. Averbeck, and H. Riechert, Appl. Phys. Lett. 76, 2122 (2000).

${ }^{5}$ J. S. Dyck, K. Kim, S. Limpijumnong, W. R. L. Lambrecht, K. Kash, and J. C. Angus, Solid State Commun. 114, 355 (2000).

${ }^{6}$ C. Persson, R. Ahuja, A. F. da Silva, and B. Johansson, J. Phys.: Condens. Matter 13, 8945 (2001).
${ }^{7}$ F. Bechstedt and J. Furthmüller, J. Cryst. Growth 246, 315 (2002).

${ }^{8}$ H. Grille, Ch. Schnittler, and F. Bechstedt, Phys. Rev. B 61, 6091 (2000).

${ }^{9}$ C. Bungaro, K. Rapcewicz, and J. Bernholc, Phys. Rev. B 61, 6720 (2000).

${ }^{10}$ S. N. Mohammad and H. Morkoc, Prog. Quantum Electron. 20, 361 (1996).

${ }^{11}$ D. C. Look, H. Lu, W. J. Schaff, J. Jasinski, and Z. Liliental-Weger, Appl. Phys. Lett. 80, 258 (2002).

${ }^{12}$ D. C. Look and J. R. Sizelove, Phys. Rev. Lett. 82, 1237 (1999).

${ }^{13}$ J. W. P. Hsu, M. J. Manfra, S. N. G. Chu, C. H. Chen, L. N. Pfeiffer, and R. J. Molnar, Appl. Phys. Lett. 78, 3980 (2001).

${ }^{14}$ T. L. Tansley and C. P. Foley, Electron. Lett. 20, 1066 (1984).

${ }^{15}$ S. Yamaguchi, M. Kariya, S. Nitta, T. Takeuchi, C. Wetzel, H. Amano, and I. Akasaki, J. Appl. Phys. 85, 7682 (1999).

${ }^{16}$ T. Inushima, V. V. Mamutin, V. A. Vekshin, S. V. Ivanov, T. Sakon, M. Motokawa, and S. Ohoya, J. Cryst. Growth 227-228, 481 (2001).

${ }^{17}$ T. Inushima, T. Shiraishi, and V. Yu. Davydov, Solid State Commun. 110, 491 (1999).

${ }^{18}$ P. Perlin, E. Litwin-Staszewska, B. Suchanek, W. Knap, J. Camassel, T. Suski, R. Piotrzkarski, I. Grzegory, S. Porowski, E. Kaminska, and J. C. Chervin, Appl. Phys. Lett. 68, 1114 (1996).

${ }^{19}$ M. C. Lee, H. C. Lin, Y. C. Pan, C. K. Shu, J. Ou, W. H. Chen, and W. K. Chen, Appl. Phys. Lett. 73, 2606 (1998).

${ }^{20}$ J. Aderhold, V. Yu. Davydov, F. Fedler, H. Klausing, D. Mistele, T. Rotter, O. Semchinova, J. Stemmer, J. Graul, J. Cryst. Growth 222, 701 (2001).

${ }^{21}$ B. B. Varga, Phys. Rev. 137, A1896 (1965).

${ }^{22}$ P. Perlin, J. Camassel, W. Knap, T. Taliercio, J. C. Chervin, T. Suski, I. Grzegory, and S. Porowski, Appl. Phys. Lett. 67, 2524 (1995).

${ }^{23}$ M. Nagai, K. Ohkawa, and M. Kuwata-Gonokami, Appl. Phys. Lett. 81, 484 (2002).

${ }^{24}$ D. B. Haddad, "Investigation of optical band gap and optical phonons in InN and $\mathrm{In}_{1-x} \mathrm{Al}_{x} \mathrm{~N}$ films," Ph.D. thesis, Wayne State University, 2003.

${ }^{25}$ A. Kasic, M. Schubert, Y. Saito, Y. Nanishi, and G. Wagner, Phys. Rev. B 65, 115206 (2002).

${ }^{26}$ J. Wu, W. Walukiewicz, W. Shan, K. M. Yu, J. W. Ager, III, E. E. Haller, H. Lu, and W. J. Schaff, Phys. Rev. B 66, 201403 (2002).

${ }^{27}$ D. B. Haddad, J. S. Thakur, V. M. Naik, G. W. Auner, R. Naik, and L. E. Wenger, Mater. Res. Soc. Symp. Proc. 743, I.11.22 (2003).

${ }^{28}$ G. W. Auner, T. D. Lenane, F. Ahmad, R. Naik, P. K. Kuo, and Z. L. Wu, in Wide Band Gap Electronic Materials (Academic, New York, 1995), p. 329.

${ }^{29}$ H. Brooks, Phys. Rev. 83, 879 (1951). 